\title{
Research Notes: Data Structures for Social Media Machine Learning - The Tweet Term Matrix (TTM) and Tweet Bio-Term Matrix (TBTM)
}

\author{
Nick V. Flor \\ Mental Systems Research Lab
}

\section{ABSTRACT}

The document term matrix ("DTM") is a representation of a collection of documents, and is a key input to many machine learning algorithms. It can be applied to a collection of tweets as well. I give the set-predicate formalism for the tweet term matrix ("TTM"), and the tweet bio-term matrix ("TBTM").

\section{INTRODUCTION}

A key challenge for Twitter text analysts is to make predictions or classifications based on a collection of tweets, usually all the tweets associated with a hashtag. Since prediction or classification algorithms process numerical input, you must find a way to represent a tweet as a vector of numbers. A document term matrix ("DTM") is a common data structure for representing a collection of text documents as a vectors of numbers. If you assume that each tweet is a kind of micro document, you can adapt the concept of a document term matrix to represent tweets as numerical vectors-a tweet term matrix ("TTM"). One can also represent meta data, such as user bios as numerical vectors-a tweet bio-term matrix ("TBTM").

\section{VARIABLE AND PREDICATE ASSUMPTIONS}

Suppose you have a dataset of tweets, $T$. Each individual tweet $\left(t_{i}\right)$ in your dataset has a corresponding user bio $\left(b_{i}\right.$, with $B$ denoting the set of all bios). Tweets and bios are strings, and Twitter limits their size to 280 and 160 characters, respectively. Formally:

$$
\begin{gathered}
\mathrm{T}=\left\{t_{i}: t_{i} \subset \text { dataset }, t_{i} \in \text { string, } 0<\left|t_{i}\right|<=280\right\} \\
\mathrm{B}=\left\{b_{i}: b_{i} \subset \text { dataset }, b_{i} \in \text { string }, 0<\left|b_{i}\right|<=160\right\} \\
T \bumpeq B
\end{gathered}
$$

We define the following predicates for operating on strings and sets of strings:

- WORDS( $x)$ : returns the set of words in a string $x$. If $x$ is a set of strings, e.g., WORDS(T), it returns a set of embedded sets of words, for all strings in $\mathrm{x}$.

- $\operatorname{FLAT}(x)$ : flattens embedded sets into a single set.

- $\operatorname{UNIQUE}(x)$ : removes duplicates in a set.

- $\operatorname{INDEX}(x, X)$ : returns the set of indices of $x$ in $X-$ used to finding occurrences of $x$ in $X$.

The appendix provides $\mathrm{R}$ implementations of WORDS, FLAT, UNIQUE, and INDEX.

\section{THE TWEET TERM MATRIX}

We can now define the tweet term matrix as follows:

$$
\begin{gathered}
Q=\left\{q_{i}: q_{i} \in \operatorname{UNIQUE}(T)\right\} \\
\left.W=\left\{w_{j}: w_{j} \in \operatorname{UNIQUE}(\operatorname{FLAT}(\operatorname{WORDS}(Q)))\right\}\right\}
\end{gathered}
$$

From $\mathrm{Q}$ and $\mathrm{W}$, one can create tweet-word matrix, $M$, where the indices of the rows correspond to the unique tweets, $Q$, and the indices of the columns corresponds to the words in $W$.

In practice it is useful to calculate the frequencies of the duplicate tweets and words, $Q F$ and $W F$, respectively:

$$
\begin{gathered}
Q F=\left\{q f_{i}: q f_{i} \in\left|\operatorname{INDEX}\left(q_{i}, T\right)\right|\right\} \\
W F=\left\{w f_{j}: w f_{j} \in\left|\operatorname{INDEX}\left(w_{j}, \operatorname{FLAT}(\operatorname{WORDS}(Q))\right)\right|\right\}
\end{gathered}
$$

Then sort $Q$ and $W$ in decreasing frequency, and only do the tweet word matrix (and subsequent predictions or classifications) on a subset of $\mathrm{Q}$ and $\mathrm{W}$.

\section{Cell Values}

We can now define the cells for any particular row $u$, and column $v$, in $M$, as follows:

$\underline{\text { Count: }}$

$$
m_{u v}=\left|\operatorname{INDEX}\left(w_{v}, \operatorname{WORDS}\left(q_{\mathrm{u}}\right)\right)\right|
$$

Binary Choice:

$$
m_{u v}=\left|\operatorname{INDEX}\left(w_{v}, \operatorname{WORDS}\left(q_{u}\right)\right)\right|>0 \rightarrow 1 \text {, otherwise } 0
$$

Term-Frequency Inverse Tweet Frequency:

A final common choice is known as the term frequency inverse-document frequency. Viewing a tweet as a kind of micro-document, we can adapt this equation to tweets. Given a specific word, $w_{v}$, and a given tweet, $q_{u}$. First, calculate the term frequency, which is how many times that word appears in the particular tweet, weighted by the total number of words in that tweet:

$$
f=\frac{\left|\operatorname{INDEX}\left(w_{v}, \operatorname{WORDS}\left(q_{u}\right)\right)\right|}{\left|\operatorname{WORDS}\left(q_{u}\right)\right|}
$$


Next, calculate how many tweets in the data set contain that word, which is the tweet frequency. Divide the number of postings by the document frequency to get the inverse document frequency-taking the log of this value:

$$
q=\log \frac{|Q|}{\left|Q \ni w_{v}\right|}
$$

The intuition is that a word that appears in all documents, is less informative, and carries less weight. Finally, the term frequency-inverse tweet frequency is:

$$
m_{u v}=f^{*} q=\frac{\left|\operatorname{INDEX}\left(w_{v}, q_{u}\right)\right|}{\left|\operatorname{WORDS}\left(q_{u}\right)\right|} \times \log \frac{|Q|}{\left|Q \ni w_{v}\right|}
$$

\section{Bio Cluster, Bio-Term Cluster}

A tweet can have many retweets, where the tweet is the same, but the users sharing the tweet are different. One can define a bio cluster for a tweet and its retweets as the collection consisting of the bio of the original tweet along with the bios of all the retweeters.

\section{Bio-Cluster}

Formally, we first obtain the set of unique tweets:

$$
Q=\left\{q_{i}: q_{i} \in \operatorname{UNIQUE}(T)\right\}
$$

Where the virality, $V$, of a tweet is its frequency in $T$, which includes the tweet and its retweets:

$$
V=\left\{v_{i}: v_{i} \in\left|\operatorname{INDEX}\left(q_{i}, T\right)\right|, 0<i<|Q|\right\}
$$

For any given tweet, $q_{i}$, its bio-cluster $(C)$, is the subset of bios that correspond with the tweet and all its retweets:

$$
C=\left\{b_{j}: j \in \operatorname{INDEX}\left(q_{i}, T\right)\right\}
$$

\section{Bio-Term Cluster}

Note that a given bio-cluster is a set of strings, with many duplicate words. The bio-term cluster is the biocluster decomposed into a set of unique words $(W)$ and a corresponding set of frequencies $(F)$. To do so, first create a flat list of all the words, including duplicates:

$$
D=\left\{d_{i}: d_{i} \in \operatorname{FLAT}(\operatorname{WORDS}(C))\right\}
$$

$D$, denotes the bio-term cluster with duplicate words, or simply the duplicate bio-term cluster. The bio-terms $(W)$ and their frequencies $(F)$, can then be defined as:

$$
\begin{gathered}
\left.W=\left\{w_{i}: w_{i} \in \operatorname{UNIQUE}(D)\right)\right\} \\
F=\left\{f_{i}: f_{i} \in\left|\operatorname{INDEX}\left(w_{i}, D\right)\right|, 0<i<|W|\right\}
\end{gathered}
$$

$W$ and $F$, together denote the bio-term cluster.
TWEeT BIO-TERM MATRIX (TBTM)

The tweet bio-term matrix is a 2-dimensional matrix where the rows indices, $u$, correspond to the unique tweets, $q_{u}$, and the column indices, $v$, correspond to the unique words, $W$, from the entire set of user bios. Given:

$$
\begin{gathered}
Q=\left\{q_{i}: q_{i} \in \operatorname{UNIQUE}(T)\right\} \\
D=\left\{d_{i}: d_{i} \in \operatorname{FLAT}(\operatorname{WORDS}(B))\right\} \\
W=\left\{w_{i}: w_{i} \in \operatorname{UNIQUE}(D)\right\} \\
F=\left\{f_{i}: f_{i} \in\left|\operatorname{INDEX}\left(w_{i}, D\right)\right|, 0<i<|W|\right\}
\end{gathered}
$$

\section{Cell Values}

We can now define the cells of the tweet bio-term matrix in terms of various heuristics, similar to the tweet term matrix.

Before doing so, it is helpful in practice to sort $Q$ and $W$ in descending frequency, and to have frequency cutoff values for both $\mathrm{Q}$ and $\mathrm{W}$, so that less viral tweets or unpopular words are not included in the calculations for the cell values.

The following heuristics all rely on first calculating the bio-cluster, $c_{u}$, and the flattened bio-cluster with all duplicate words, $d_{u}$, for a given row, $u$ :

$$
\begin{gathered}
c_{u}=\left\{b_{k}: k \in \operatorname{INDEX}\left(q_{u}, T\right)\right\} \\
d_{u}=\left\{\operatorname{FLAT}\left(\operatorname{WORDS}\left(c_{u}\right)\right)\right\}
\end{gathered}
$$

\section{Binary Heuristic}

$$
m_{u v}=\left|\operatorname{INDEX}\left(w_{v}, \mathrm{~d}_{u}\right)\right|>0 \rightarrow 1: 0
$$

\section{Count Heuristic}

$$
m_{u v}=\left|\operatorname{INDEX}\left(w_{v}, \mathrm{~d}_{u}\right)\right|
$$

$\underline{\text { Frequency Heuristic }}$

$$
m_{u v}=\frac{\left|\operatorname{INDEX}\left(w_{v}, d_{u}\right)\right|}{\left|d_{u}\right|}
$$

Term Frequency - Inverse Bio Frequency

A final common cell choice is based on the algorithm for calculating term frequency-inverse document frequency (TF-IDF), except bio frequency substitutes for document frequency. Given a specific word, $w_{v}$, and a duplicate bioterm cluster $d_{u}$. First, calculate the term frequency, which is how many times that word appears in the duplicate bio-term cluster, weighted by the total number of words in the duplicate bio-term cluster:

$$
f=\frac{\left|\operatorname{INDEX}\left(w_{v}, d_{u}\right)\right|}{\left|d_{u}\right|}
$$


Next, calculate how many bios in the data set contain that word, which is the bio frequency. Divide the number of bios by the bio frequency to get the inverse bio frequency-taking the log of this value:

$$
q=\log \frac{|B|}{\left|B \ni w_{v}\right|}
$$

Finally:

$$
m_{u v}=f^{*} q=\frac{\left|\operatorname{INDEX}\left(w_{v}, d_{u}\right)\right|}{\left|\mathrm{d}_{u}\right|} \times \log \frac{|B|}{\left|B \ni w_{v}\right|}
$$

\section{APPENDIX: R IMPLEMENTATION OF PREDICATES AND OTHER IMPLEMENTATION DETAILS}

$\begin{array}{llll}\text { WORDS } & =\operatorname{function}(x) & \{\operatorname{strsplit}(x, \cdots) & \text { " } \\ \text { INDEX } & =\operatorname{function}(x, x) & \{\operatorname{which}(X=x) & \} \\ \text { FLAT } & =\operatorname{function}(x) & \{\operatorname{unlist}(x) & \} \\ \text { UNIQUE }=\text { function }(x) & \{\operatorname{unique}(x) & \}\end{array}$

Finally, one can implement expressions with the syntax $|\mathrm{x}|$ as $\operatorname{length}(\mathrm{x})$, and $B \ni w_{v}$ as $\operatorname{grep}\left(w_{v}, B\right)$.

It is also useful to first pre-process tweets and bios using the following cleaning function:

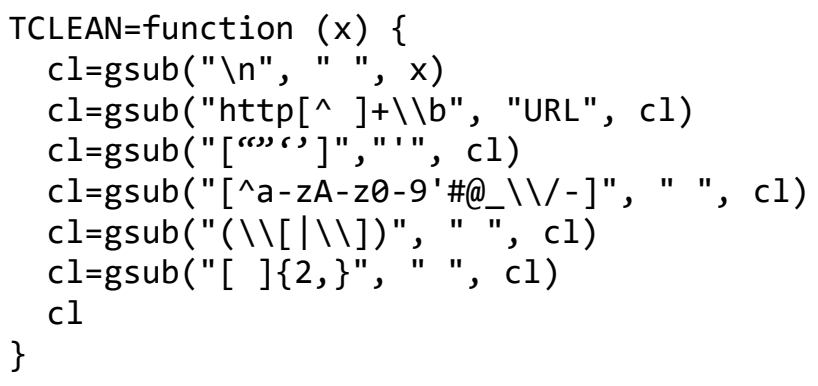

It can also be helpful to pre-process any words, which includes removing stop words and small words:

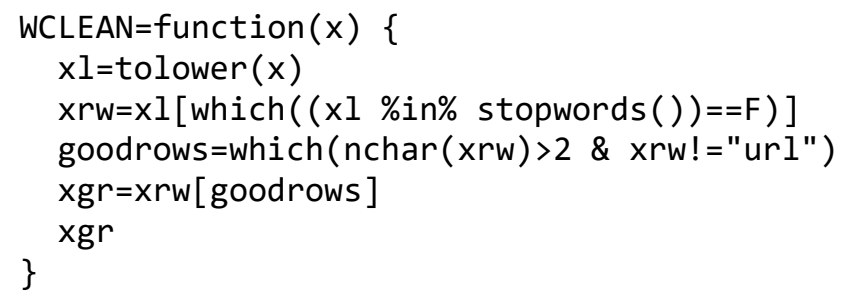

Lastly, $T$ and $B$ depend on which package one uses in $\mathrm{R}$ to scrape tweets. The package rtweet is a popular one for scraping tweets. Using rtweet, $T$ and $B$ would be implemented as follows:

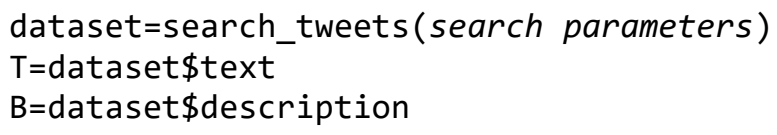

